\title{
Noise-induced complex oscillatory dynamics in the Zeldovich-Semenov model of a continuous stirred tank reactor
}

\author{
Cite as: Chaos 31, 013105 (2021); doi: 10.1063/5.003051 1 \\ Submitted: 22 September 2020 . Accepted: 10 December 2020 . \\ Published Online: 4 January 2021
}

Lev Ryashko

\author{
AFFILIATIONS \\ Ural Mathematical Center, Ural Federal University, Lenina, 51, 620000 Ekaterinburg, Russia
}

Note: This paper belongs to the Focus Issue, Recent Advances in Modeling Complex Systems: Theory and Applications.

${ }^{a}$ Author to whom correspondence should be addressed: lev.ryashko@urfu.ru

\begin{abstract}
Noise-induced variability of thermochemical processes in a continuous stirred tank reactor is studied on the basis of the Zeldovich-Semenov dynamical model. For the deterministic variant of this model, mono- and bistability parametric zones as well as local and global bifurcations are determined. Noise-induced transitions between coexisting attractors (equilibria and cycles) and stochastic excitement with spike oscillations are investigated by direct numerical simulation and the analytical approach based on the stochastic sensitivity technique. For the stochastic model, the phenomenon of coherence resonance is discovered and studied.
\end{abstract}

Published under license by AIP Publishing. https://doi.org/10.1063/5.0030511

In nonlinear dynamics, an approach has been formed in which the investigation of complex modes of behavior of real systems is reduced to the study of bifurcations, transformations of attractors, basins of attraction, and separatrices in the corresponding mathematical models. It is well known that combustion processes are characterized by a variety of dynamic modes with abrupt transitions from simple equilibrium regimes to complex oscillatory ones. Among mathematical models of thermochemical processes, the Zeldovich-Semenov (ZS) model, which describes the dynamics of an exothermic reaction in a continuous stirred tank reactor (CSTR), is well known. This model demonstrates mono- and bistability as well as local and global bifurcations. The aim of the paper is to show how the inevitable, even small, random noise significantly intricates the already complex deterministic dynamics of this model. For quantitative analysis and prognosis of noise-induced phenomena, we use the stochastic sensitivity apparatus.

\section{INTRODUCTION}

A continuous stirred tank reactor (CSTR) is one of the key objects of chemical engineering that allows one to study the fundamental mechanisms of basic dynamical modes in combustion processes. $^{1-4}$ For a description and analysis of thermochemical reactions in such reactors, diverse mathematical models were elaborated and studied. ${ }^{5-10}$ An important distinctive feature of thermochemical kinetics is its high sensitivity to changes in parameters. Even very small variations in the kinetic parameters can lead to significant transformations of the dynamical regimes of the reactor.

In the investigation of complex CSTR dynamics, quite a large number of studies have been devoted to the Zeldovich-Semenov (ZS) model (see, i.e., Refs. 6 and 11-13). This basic model of the thermal explosion theory describes the dynamics of an exothermic reaction in a CSTR. ${ }^{2,14,15}$ In the course of the studies of the deterministic ZS-model, it was shown that the experimentally observed complexity of the behavior of kinetic processes in the reactor is associated with an amazing variety of possible types of phase portraits changing through the local and global bifurcations. Here, one of the main reasons of the complexity of kinetic regimes is multistability. Due to the coexistence of several different type attractors (equilibria, cycles), seemingly insignificant disturbances can lead to sharp jumps in temperature and concentration with the formation of new unexpected regimes.

The purpose of this paper is to show how even small random perturbations that inevitably accompany the CSTR operation can abruptly change dynamic regimes and cause transitions 
between attractors with the generation of complex multimodal spike oscillations. In this paper, on the base of ZS-model, we suggest a constructive approach to probabilistic analysis of noise-induced phenomena, which complicate the overall picture of the kinetics of thermochemical processes in CSTR.

Noise-induced deformations of dynamic modes in nonlinear systems attract the attention of researchers from various fields of natural science. The mutual influence of nonlinearity and stochasticity gives rise to such phenomena as noise-induced transitions, ${ }^{16}$ stochastic and coherent resonances, ${ }^{17-20}$ stochastic excitability, ${ }^{21}$ noise-induced chaos-order transformations, ${ }^{22-24}$ stochastic bifurcations, ${ }^{25}$ noise-induced complexity, ${ }^{26}$ etc. These phenomena were discovered by methods of direct numerical simulation of solutions of the corresponding stochastic models. However, the study of underlying probabilistic mechanisms and the extended parametric investigation of these phenomena require analytical approaches. Nowadays, methods based on the various asymptotics and approximations are in demand. ${ }^{27,28}$ Among them, the stochastic sensitivity function technique is effectively used. ${ }^{29-32}$ The apparatus of confidence domains elaborated on the basis of this technique turned out to be useful in the analysis of complex stochastic effects in nonlinear dynamical systems. ${ }^{33-38}$ In the present paper, we show how confidence domains method can be applied to the parametric analysis of various noise-induced phenomena in the Zeldovich-Semenov dynamical model of CSTR.

In Sec. II, we present the results of the analysis of the deterministic ZS-model in the parametric zone with the local Andronov-Hopf bifurcation and global bifurcation of the merging of the cycle and stable manifold of the saddle point. Here, various regimes of bi- and monostability of the ZS-model are described that are important for the study of stochastic dynamics.

In Sec. III, we consider the ZS-model with random disturbances. Here, in bistability zones, noise-induced transitions "equilibrium $\leftrightarrow$ equilibrium" and "equilibrium $\leftrightarrow$ cycle" are studied. In the monostability zone, where the deterministic ZS-model exhibits the equilibrium as the only attractor, we analyze the phenomenon of the stochastic excitement with spike oscillations.

Section IV is devoted to the constructive analysis of underlying probabilistic mechanisms of these phenomena by the stochastic sensitivity technique.

a)

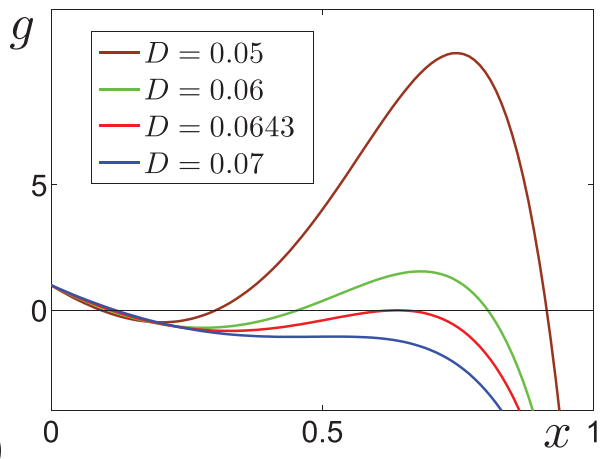

In Sec. V, statistics of interspike intervals (ISIs) in the zone of stochastic excitability are studied and the phenomenon of coherence resonance is discussed.

\section{DETERMINISTIC MODEL}

The deterministic variant of the conceptual ZeldovichSemenov dynamical CSTR model is usually written ${ }^{10}$ as

$$
\begin{aligned}
& \dot{x}=(1-x) \exp \left(\frac{y}{1+\beta y}\right)-\frac{x}{D}, \\
& \gamma \dot{y}=(1-x) \exp \left(\frac{y}{1+\beta y}\right)-\frac{y}{S} .
\end{aligned}
$$

Here, dimensionless variables $x$ and $y$ stand for the concentration and temperature, respectively, and $\beta, \gamma, S$, and $D$ are dimensionless parameters. Under the variation of parameters, this system exhibits a lot of diverse dynamical regimes. In our study, we fix $\beta=0.05$, $\gamma=0.03$, and $S=0.4$, and use $D$ as a control parameter. Even within these constraints, this model allows us to cover the cases of mono- and bistability with attractors in the form of equilibria and cycles undergoing both local and global bifurcations.

Coordinates of equilibria of system (1) can be found from equations

$$
g(x)=(1-x) \exp \left(\frac{S x}{D+\beta S x}\right)-\frac{x}{D}=0, \quad y=\frac{S}{D} x .
$$

Plots of the function $g(x)$ are shown in Fig. 1(a) for various values of $D$. In Fig. 1(b), $x$-coordinates of these equilibria are plotted for $0.04 \leq D \leq 0.1$. Here, for any $D$, system (1) has the stable equilibrium $L$ (green). This equilibrium is unique for $D>D_{3}=0.0643$. As $D$ passes $D_{3}$ from the right to left, in system (1), two equilibria appear: $H$ (blue) and $U$ (red). The bifurcation point $D_{3}$ was found from the condition that the curve $g(x)$ touches the line $y=0$.

The equilibrium $U$ is unstable for any $D<D_{3}$, whereas the equilibrium $H$ is unstable only in the subinterval $D_{1}<D<D_{3}$. The equilibrium $H$ is stable for $D<D_{1}$. Note that $D_{1}=0.0494$ is the Andronov-Hopf bifurcation point. This bifurcation point $D_{1}$ was found from the condition that eigenvalues of the Jacobi matrix

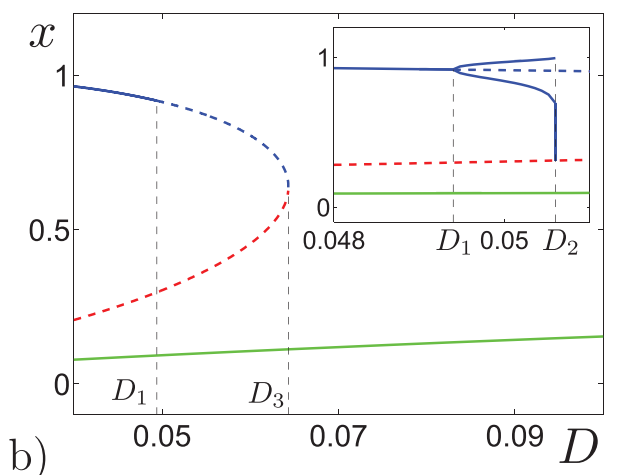

FIG. 1. Deterministic system (1): (a) plots of the function $g(x)$ and (b) bifurcation diagram. Here, bifurcation points are $D_{1}=0.0494, D_{2}=0.05063128$, and $D_{3}=0.0643$. 
a)
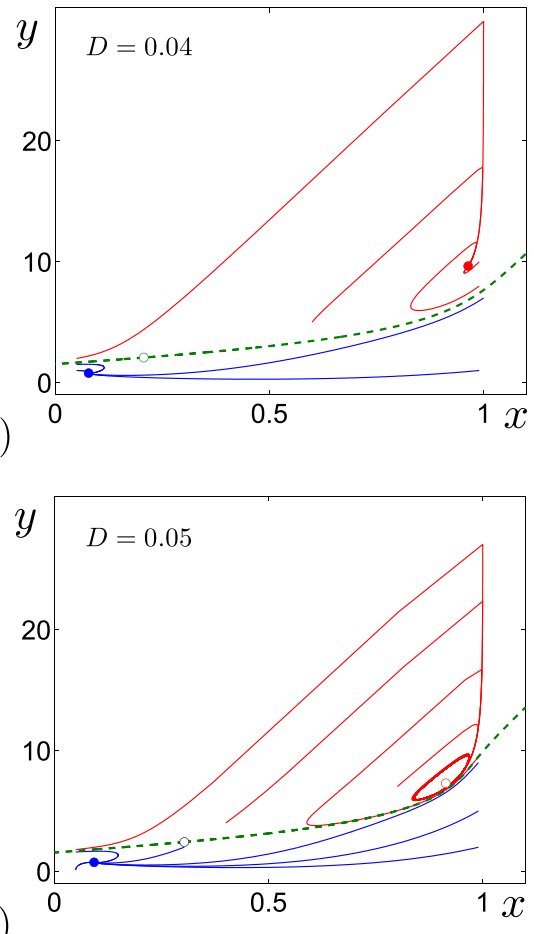

c)

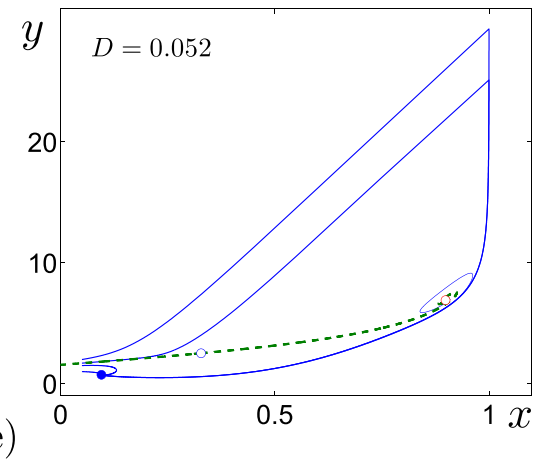

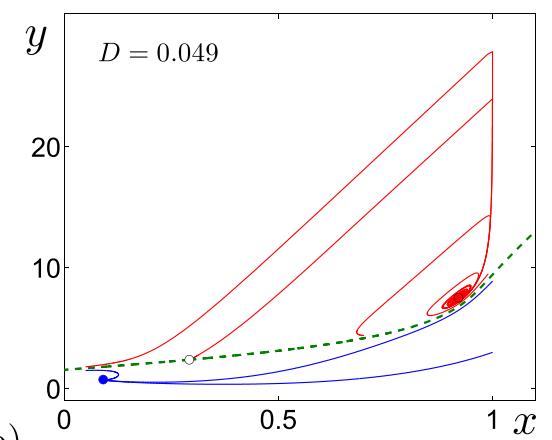

b)

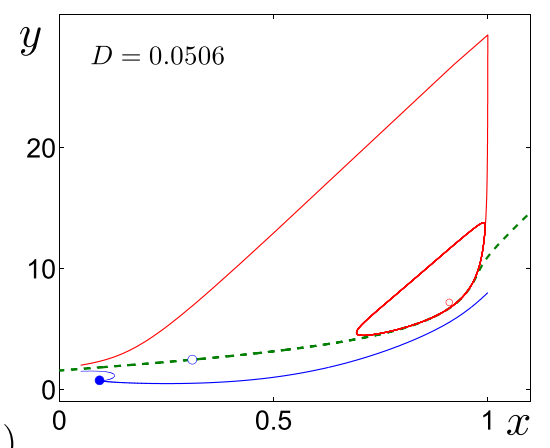

d)

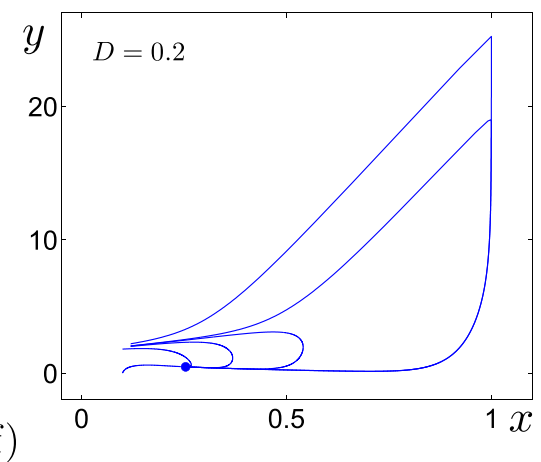

FIG. 2. Phase portraits of the deterministic system (1) for: (a) $D=0.04$, (b) $D=0.049$, (c) $D=0.05$, (d) $D=0.0506$, (e) $D=0.052$, and (f) $D=0.2$. Here, stable and unstable equilibria are shown by filled and empty circles correspondingly. The separatrix is plotted by the dashed line. of system (1) at the equilibrium $H$ have zero real parts. In the subinterval $D_{1}<D<D_{2}, D_{2}=0.05063128$, system (1) exhibits a stable limit cycle $C$ around the unstable equilibrium $H$. At the bifurcation point $D_{2}$, cycle $C$ merges with the stable invariant manifold of the saddle equilibrium $U$. The bifurcation point $D_{2}$ was found numerically.

So, changes in the deterministic dynamics are connected with local bifurcations at the points $D_{1}$ and $D_{3}$, and the global bifurcation at $D_{2}$. For $D<D_{1}$, system (1) is bistable with coexisting stable equilibria $L$ and $H$. For $D_{1}<D<D_{2}$, system (1) is bistable with coexisting stable equilibrium $L$ and stable limit cycle $C$. For $D>D_{2}$, the equilibrium $L$ is a single attractor of system (1).

In Fig. 2, we show characteristic phase portraits of system (1) for various values of $D$. Here, we point out specific deterministic features that are important for understanding stochastic phenomena discussed in Sec. III. In Fig. 2, the low-temperature equilibrium
$L$ is plotted by the blue filled circle, and phase trajectories attracted by $L$ are also shown in blue. The high-temperature attractors, the equilibrium $H$ or the cycle $C$, are plotted by the red filled circle or the thick red curve, correspondingly. Phase trajectories attracted by the "hot" attractor are shown in red. Unstable equilibria are plotted by empty circles. The green dashed curve $S$ shows the stable manifold of the unstable saddle equilibrium $U$. This manifold plays an important role in the behavior of the system (1): the curve $S$ serves as a separatrix between basins of low-temperature equilibrium $L$ and a "hot" attractor $(H$ or $C)$.

As can be seen, in the bistability regime [see Figs. 2(a)-2(d)], the location of the equilibrium $L$ and behavior of phase trajectories lying in its basin change insignificantly. As for the "hot" attractor, its position and shape change crucially. Indeed, as parameter $D$ increases, this attractor approaches separatrix $S$ and transforms from the equilibrium $H$ into the enlarging cycle $C$. 
Despite the fairly large distance of the coexisting attractors from each other, transitions between them can occur even for small disturbances. This phenomenon is explained by the closeness of these attractors to the separatrix $S$ dividing their basins.

As the parameter $D$ passes the bifurcation value $D_{2}$ from left to right, the limit cycle $C$ touches the separatrix $S$ and disappears, so the system becomes monostable. Figure $2($ e) shows for $D=0.052$ the case with one stable $(L)$ and two unstable ( $U$ and $H$ ) equilibria, and Fig. 2(f) gives an example where the equilibrium $L$ is the only one.

Another important common feature of phase portraits is the following. The transient processes of solutions starting from points lying above the equilibrium $L$ at a distance exceeding a certain critical level demonstrate a sharp large-amplitude blowout to the right and upward, followed by the downward tending to its attractor.

In Sec. III, we will show how these peculiarities of the deterministic model complicate the behavior of the system in presence of random disturbances.

\section{STOCHASTIC MODEL}

In the analysis of noise-induced effects, we will consider the following stochastic model:

$$
\begin{aligned}
& \dot{x}=(1-x) \exp \left(\frac{y}{1+\beta y}\right)-\frac{x}{D}, \\
& \gamma \dot{y}=(1-x) \exp \left(\frac{y}{1+\beta y}\right)-\frac{y}{S}+\varepsilon \xi .
\end{aligned}
$$

Here, $\xi(t)$ is uncorrelated standard white Gaussian noise with parameters $\mathrm{E} \xi(t)=0, \mathrm{E} \xi(t) \xi(\tau)=\delta(t-\tau)$, and $\varepsilon$ is the noise intensity.

Consider what new dynamical regimes can appear in the CSTR model (1) in the presence of random disturbances.

First, let $D=0.04$. In the upper panel of Fig. 3, phase trajectories and $y$-coordinates of time series of system (2) solutions starting from the stable deterministic equilibrium $L$ are plotted for three values of the noise intensity.

For weak noise $(\varepsilon=0.03$, green), random trajectories fluctuate near the equilibrium $L$. Under increasing noise, dispersion of random trajectories grows, and solutions of system (2) cross the separatrix $S$, fall into the basin of the equilibrium $H$, and continue small-amplitude oscillations near $H$ after the sharp blowout [see blue trajectory for $\varepsilon=0.1$ in Figs. 3(a) and 3(b)]. With further increase of noise, the opposite transitions from $H$ to $L$ occur. As a result, system (2) shows the alternation of oscillations near $L$ and $H$ with spike transients (see red trajectories for $\varepsilon=0.5$ ).

Details of such noise-induced transformations can be seen in the lower panel of Fig. 3 where changes of the random states distribution vs noise intensity $\varepsilon$ are demonstrated. Here, states of random solutions starting from the low-temperature equilibrium $L$ [Fig. 3(c)] and the hot equilibrium $H$ [Fig. 3(d)] are shown after the transient process.

In Fig. 3(c), three stages can be distinguished. At the first stage, for small noise, trajectories concentrate near the equilibrium $L$. The second stage reflects the transition from $L$ to $H$ for larger noise intensities. At the third stage, the system exhibits complex mixed-mode stochastic oscillations with blowouts of large amplitudes.

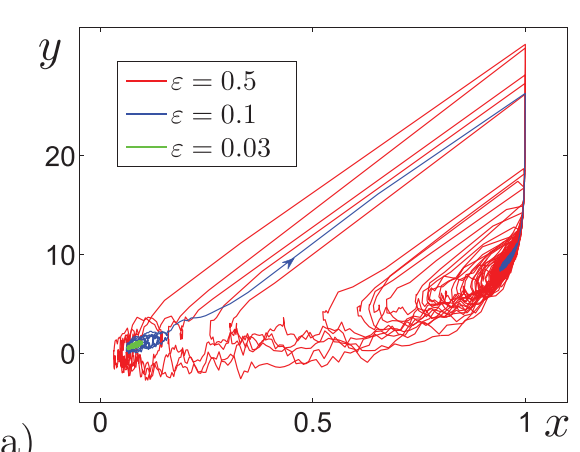

a)

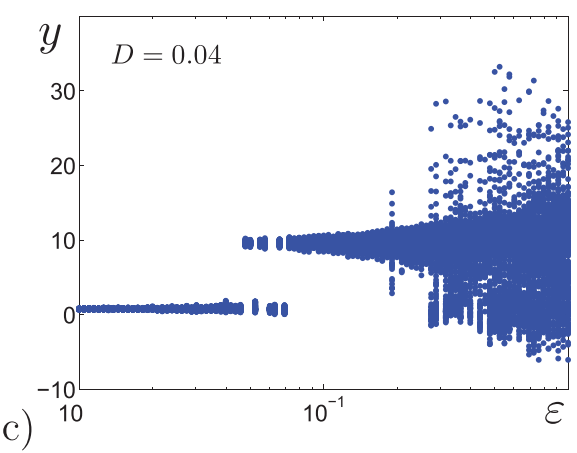

b)

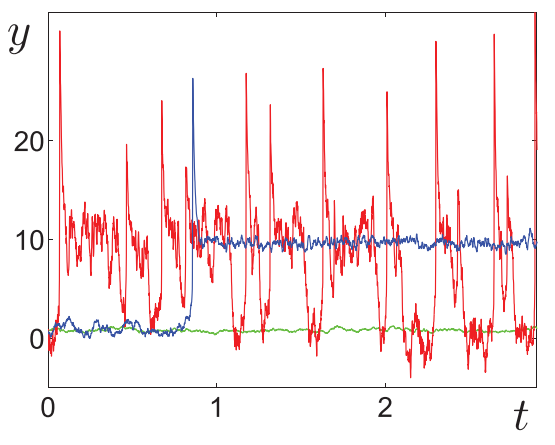

d)

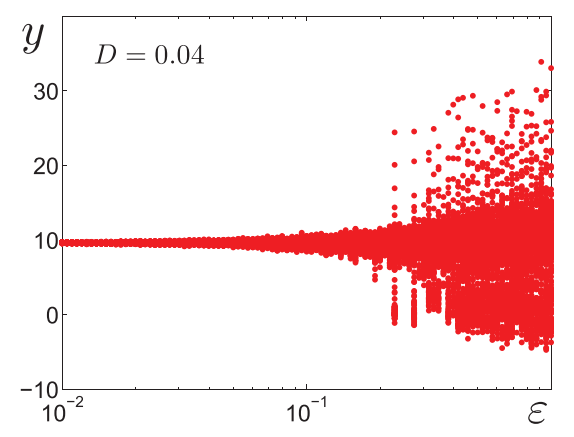

FIG. 3. Stochastic system (2) with $D=0.04$ : (a) phase trajectories and (b) time series for three values of the noise intensity; $y$-coordinates of random states for solutions starting from (c) equilibrium $L$ and (d) equilibrium $H$ vs noise intensity $\varepsilon$. 
In Fig. 3(d), one can see only two stages: small-amplitude oscillations near $H$ and mixed-mode oscillations with transitions between basins of $L$ and $H$.

Consider how these scenarios of the transformation of noiseinduced dynamics change under increasing parameter D. In Fig. 4, stochastic transformations are shown for $D=0.049$. In the upper panel, phase trajectories and $y$-coordinates of time series of system (2) solutions starting from the hot equilibrium $H$ are plotted. For $\varepsilon=0.01$, these trajectories (green) are localized near $H$, for $\varepsilon=0.05$, the system solutions (blue) transit from $H$ to $L$, and for $\varepsilon=0.1$, the mixed-mode oscillations (red) with transitions between $H$ and $L$ are observed. The lower panel shows deformations of the distributions of random solutions starting from $L$ [Fig. 4(c)] and from $H$ [Fig. 4(d)]. Here, one can see two-stage scenario in Fig. 4(c) and three-stage scenario in Fig. 4(d).

Comparing Fig. 3 with Fig. 4, one can resume that in the system with $D=0.04$, some kind of the "stochastic preference" of $H$ is observed, whereas for $D=0.049$, equilibrium $L$ dominates.

In Fig. 5, noise-induced transitions are shown for $D=0.0506$ where the deterministic system possesses bistability with the coexistence of the stable equilibrium $L$ and stable cycle $C$. In the upper panel, phase trajectories of system (2) solutions starting from cycle $C$ are plotted for three values of the noise intensity. For $\varepsilon=0.0005$, these trajectories (green) are localized near the orbit of $C$ for larger noise with $\varepsilon=0.03$, the system solutions (blue) transit from $C$ to $L$, and for $\varepsilon=0.1$, the mixed-mode oscillations (red) with transitions between $C$ and $L$ can be seen. This is a birhythmic regime with the alternation of oscillations near $C$ and spike transient oscillations between $C$ and $L$. Here, the lower-temperature equilibrium stochastically dominates again [see Figs. 5(c) and 5(d)].
We now turn to the study of stochastic effects in the monostability zone, where the system exhibits the stable equilibrium $L$ as the only attractor. In Fig. 6(a), for $D=0.052$, time series of solutions starting from $L$ are plotted for two values of the noise intensity. Here, the phenomenon of noise-induced excitement is observed. Indeed, for small noise $\varepsilon=0.05$, the system exhibits small-amplitude random fluctuations near $L$, whereas for $\varepsilon=0.1$, spikes appear. It can be seen that in some spikes the system slows down and makes a turnover near the unstable equilibrium $H$. In Fig. 6(c), the sharp transition to noise-induced excitement is illustrated for gradually increasing $\varepsilon$.

Similar scenario is observed for $D=0.2$ (right panel of Fig. 6) the onset of the spiking oscillations occurs for larger $\varepsilon$.

Numerical results presented above for the stochastic CSTR model show a variability of noise-induced effects with complex oscillatory behavior. For the parametric study of these phenomena, the analytical approach based on the stochastic sensitivity technique and confidence domains method can be applied.

\section{STOCHASTIC SENSITIVITY ANALYSIS}

As shown in Secs. I-III, in the analysis of noise-induced phenomena in multistable and excitable systems, the remoteness of the attractor from the separatrix plays an important role. However, along with this fact, one has to take into account sensitivity of attractors to noise. Indeed, for different attractors, the same noise can result in the different dispersion of random states. The stochastic sensitivity function is a measure of the response of the attractor to weak noise. a)
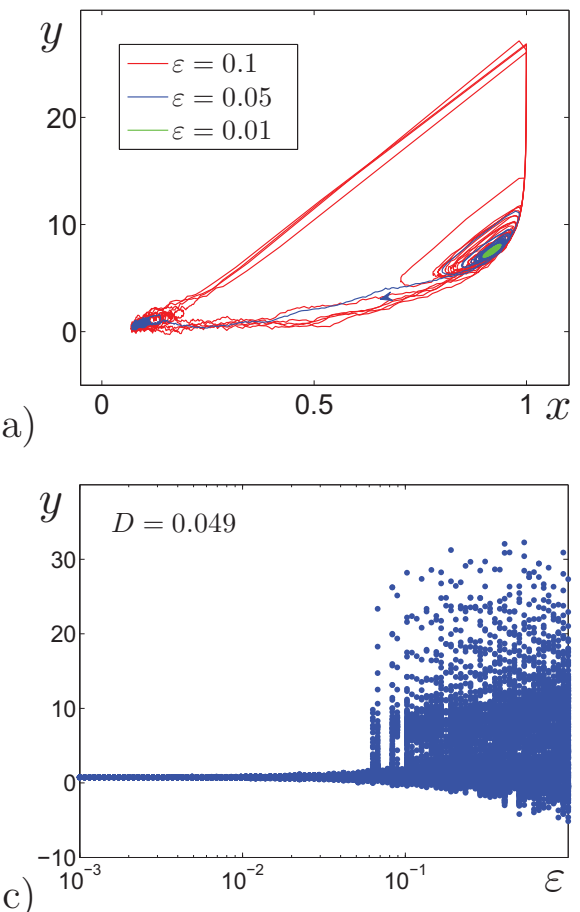

b)

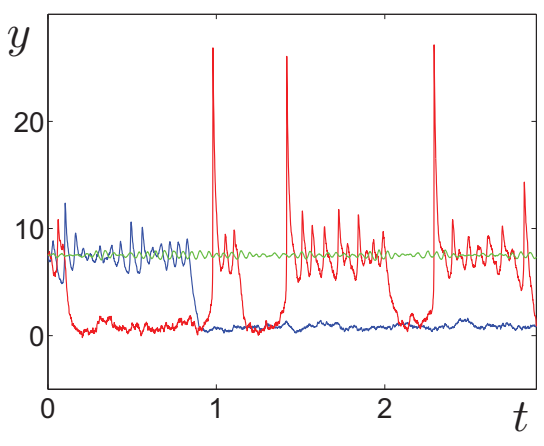

d)

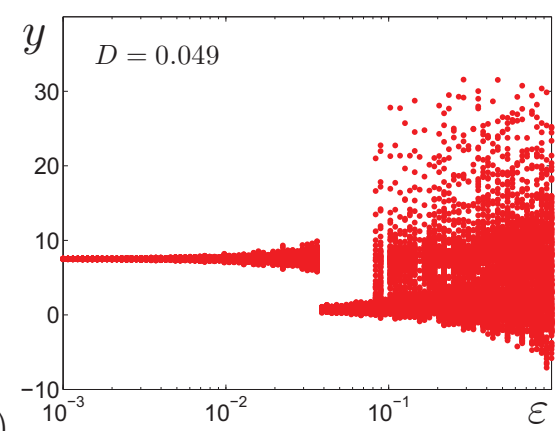

FIG. 4. Stochastic system (2) with $D=0.049$ : (a) phase trajectories and (b) time series for three values of the noise intensity; $y$-coordinates of random states for solutions starting from (c) equilibrium $L$ and (d) equilibrium $H$ vs noise intensity $\varepsilon$. 
a)
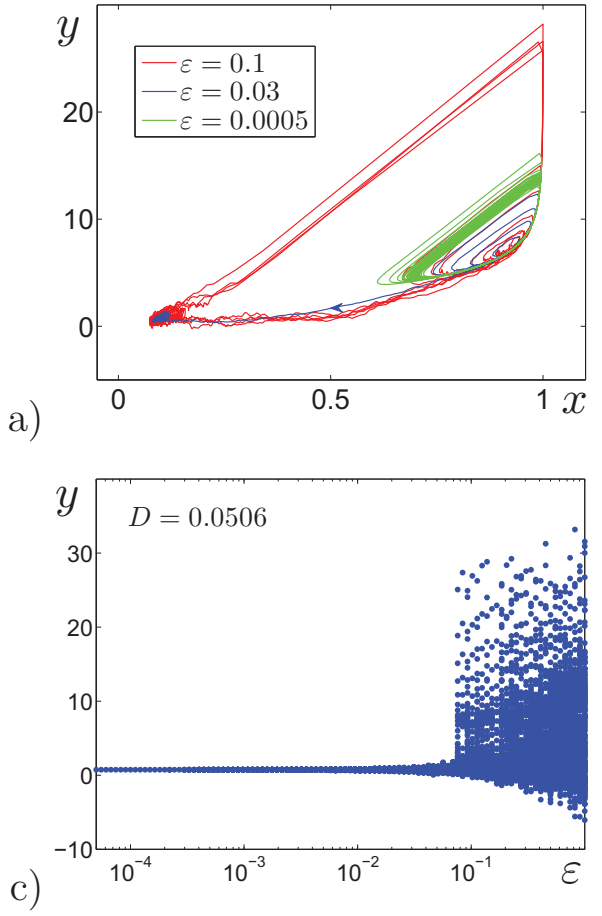

b)
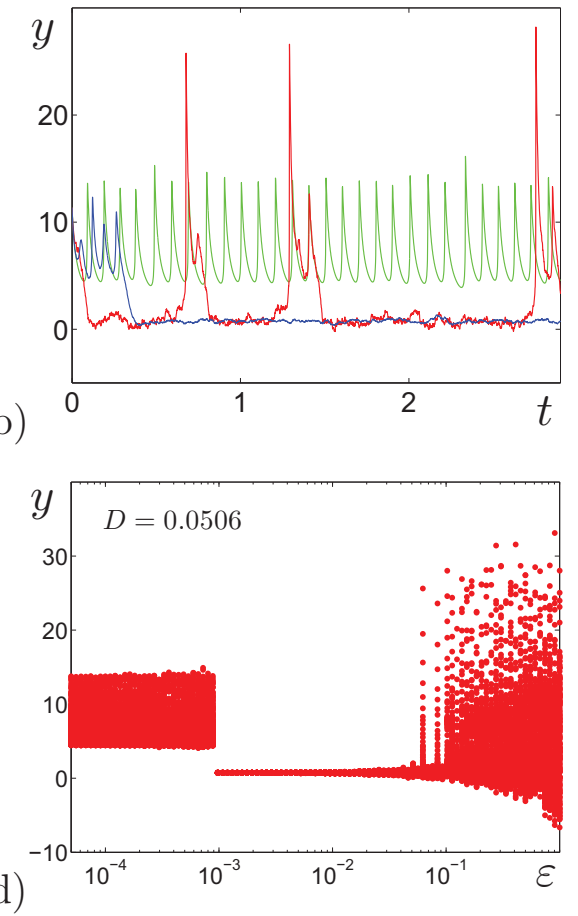

FIG. 5. Stochastic system (2) with $D=0.0506$ : (a) phase trajectories and (b) time series for three values of the noise intensity; $y$-coordinates of random states for solutions starting from (c) equilibrium $L$ and (d) cycle $C$ vs noise intensity $\varepsilon$.
The quantitative characteristic of the sensitivity of a stable equilibrium to random disturbances is the stochastic sensitivity matrix $W .^{36}$

Indeed, for the general system

$$
\dot{\mathbf{x}}=\mathbf{f}(\mathbf{x})+\varepsilon \sigma(\mathbf{x}) \xi(t)
$$

where $\xi(t)$ is a standard white Gaussian noise with $\mathrm{E} \xi(t)$ $=0, \mathrm{E} \xi(t) \xi^{\top}(\tau)=\delta(t-\tau) I$, and $\varepsilon$ is the noise intensity, and one can find the following Gaussian approximation of the probability density function $\rho(\mathbf{x}, \varepsilon)$ in a neighborhood of the equilibrium $\overline{\mathbf{x}}$ :

$$
\rho(\mathbf{x}, \varepsilon) \approx N \exp \left(-\frac{(\mathbf{x}-\overline{\mathbf{x}})^{\top} W^{-1}(\mathbf{x}-\overline{\mathbf{x}})}{2 \varepsilon^{2}}\right)
$$

with the mean $\overline{\mathbf{x}}$, the covariance matrix $C=\varepsilon^{2} W$, and the normalization constant, $N$.

The positive definite symmetric matrix $W$ is a unique solution of the matrix Lyapunov equation,

$$
J W+W J^{\top}+Q=0, \quad J=\frac{\partial \mathbf{f}}{\partial \mathbf{x}}(\overline{\mathbf{x}}), \quad Q=\sigma(\overline{\mathbf{x}}) \sigma^{\top}(\overline{\mathbf{x}}) .
$$

For the CSTR model (2) considered here,

$$
\begin{aligned}
& J=\left[\begin{array}{cc}
-\exp \left(\frac{y}{1+\beta y}\right)-\frac{1}{D} & \frac{(1-x)}{(1+\beta y)^{2}} \exp \left(\frac{y}{1+\beta y}\right) \\
-\frac{1}{\gamma} \exp \left(\frac{y}{1+\beta y}\right) & \frac{(1-x)}{\gamma(1+\beta y)^{2}} \exp \left(\frac{y}{1+\beta y}\right)-\frac{1}{\gamma S}
\end{array}\right], \\
& Q=\left[\begin{array}{cc}
0 & 0 \\
0 & \frac{1}{\gamma^{2}}
\end{array}\right] .
\end{aligned}
$$

Eigenvectors $v_{1}, v_{2}$ and eigenvalues $\lambda_{1}, \lambda_{2}$ of the stochastic sensitivity matrix $W$ define the directions of axes and a size of the confidence ellipse,

$$
\frac{z_{1}^{2}}{\lambda_{1}}+\frac{z_{2}^{2}}{\lambda_{2}}=-2 \varepsilon^{2} \ln (1-P)
$$

Here, $z_{1}, z_{2}$ are coordinates of this ellipse in the basis of $v_{1}, v_{2}$ with the equilibrium as the origin, $\varepsilon$ is the noise intensity, and $P$ is the fiducial probability. In our study, we use $P=0.999$.

In a two-dimensional system, the stochastic sensitivity function $\mu(t)$ of the stable limit cycle $C$ is a unique $T$-periodic solution of the boundary problem, ${ }^{24,36}$

$$
\dot{\mu}=a(t) \mu+b(t), \quad \mu(0)=\mu(T),
$$

with coefficients

$$
a(t)=p^{\top}(t)\left(J^{\top}(t)+J(t)\right) p(t), \quad b(t)=p^{\top}(t) Q p(t) .
$$



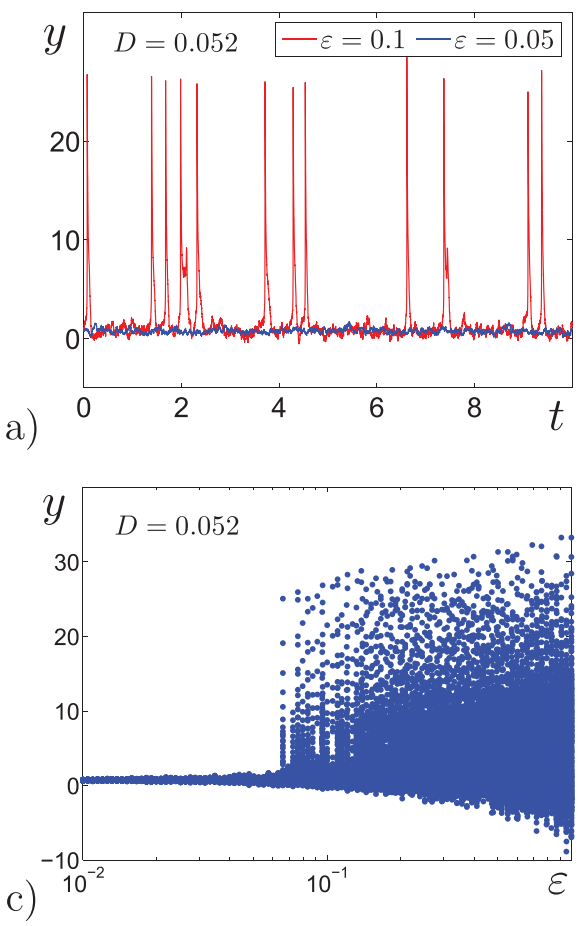
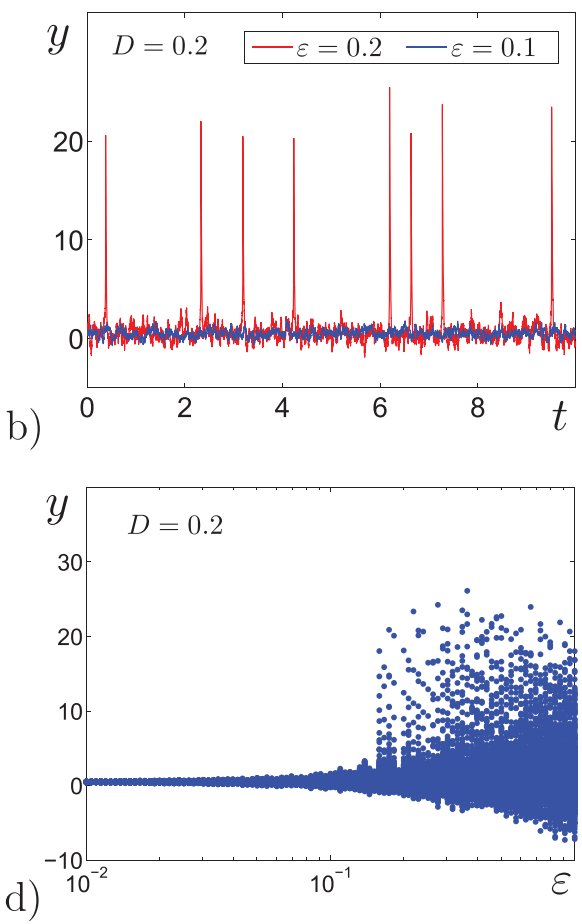

FIG. 6. Stochastic system (2): time series (a) for $D=0.052$ and (b) for $D=0.2$; $y$-coordinates of random states for solutions starting from the equilibrium $L$ for $(c)$ $D=0.052$ and (d) $D=0.2$.
Here, the Jacobi matrix $J(t)$ is calculated at the current point $(\bar{x}(t), \bar{y}(t))$ of the cycle, the matrix $Q$ for system (2) is defined above, and $p(t)=\left(p_{x}(t), p_{y}(t)\right)^{\top}$ is a normalized vector that is orthogonal to the cycle $C$ at the current point.

Using the function $\mu(t)$, one can find borders of the confidence band around the limit cycle $C$ as follows:

$$
\left(\begin{array}{l}
x_{1,2}(t) \\
y_{1,2}(t)
\end{array}\right)=\left(\begin{array}{c}
\bar{x}(t) \\
\bar{y}(t)
\end{array}\right) \pm k \varepsilon \sqrt{2 \mu(t)}\left(\begin{array}{c}
p_{x}(t) \\
p_{y}(t)
\end{array}\right), t \in[0, T] .
$$

Here, $k=\operatorname{erf}^{-1}(P), \operatorname{erf}(x)=\frac{2}{\sqrt{\pi}} \int_{0}^{x} e^{-t^{2}} d t$, and $P$ is the fiducial probability.

In Fig. 7(a), plots of eigenvalues $\lambda_{1}(D)$ and $\lambda_{2}(D)$ of stochastic sensitivity matrices $W(D)$ for equilibria $L$ and $H$ are shown in blue and red, respectively. As can be seen, the stochastic sensitivity of the equilibria differs and depends on $D$ in different ways.
In Fig. $7(\mathrm{~b})$, plots of the stochastic sensitivity function $\mu(t)$ are shown in $D$-parameter zone where the deterministic system (1) possesses stable limit cycles. As one can see, the stochastic sensitivity is highly non-uniform along the cycle.

Figures 8 and 9 summarize results of our stochastic sensitivity analysis of noise-induced phenomena considered above.

In Fig. $8(\mathrm{a})$, for $D=0.04$, we show equilibria $L$ and $H$, separatrix $S$, and confidence ellipses for three values of the noise intensity $\varepsilon$. Parameters $D$ and $\varepsilon$ are the same as in Fig. 3(a). For $\varepsilon=0.03$, confidence ellipses totally belong to the corresponding basins of attraction. This confirms that random solutions starting from equilibria are localized near these initial states. For $\varepsilon=0.1$, the confidence ellipse around $H$ is still in the basin of $H$, whereas the confidence ellipse around $L$ intersects the separatrix $S$ (green dashed) and partially occupies points of the basin of $H$. Such an a)

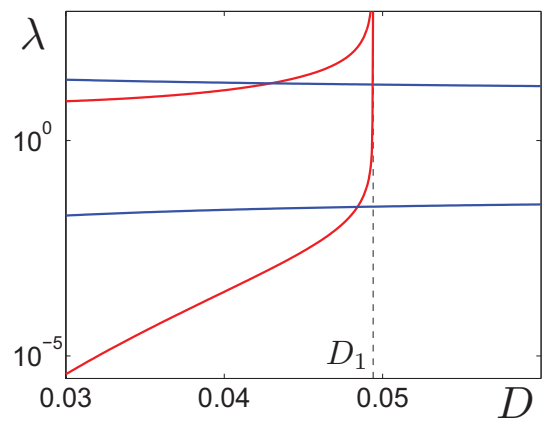

b)

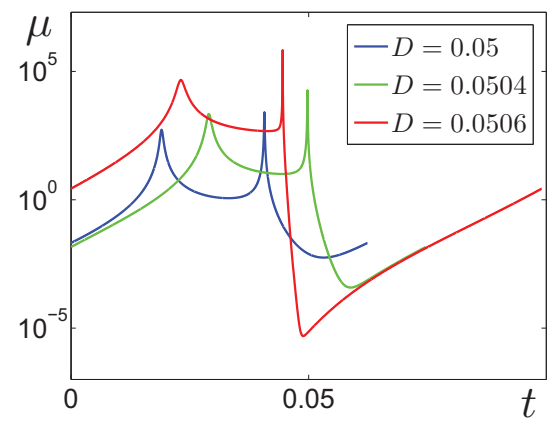

FIG. 7. Stochastic sensitivity of attractors of system (2): (a) eigenvalues of the stochastic sensitivity matrices for the equilibria $L$ (blue) and $H$ (red) and (b) stochastic sensitivity of cycles. 
a)
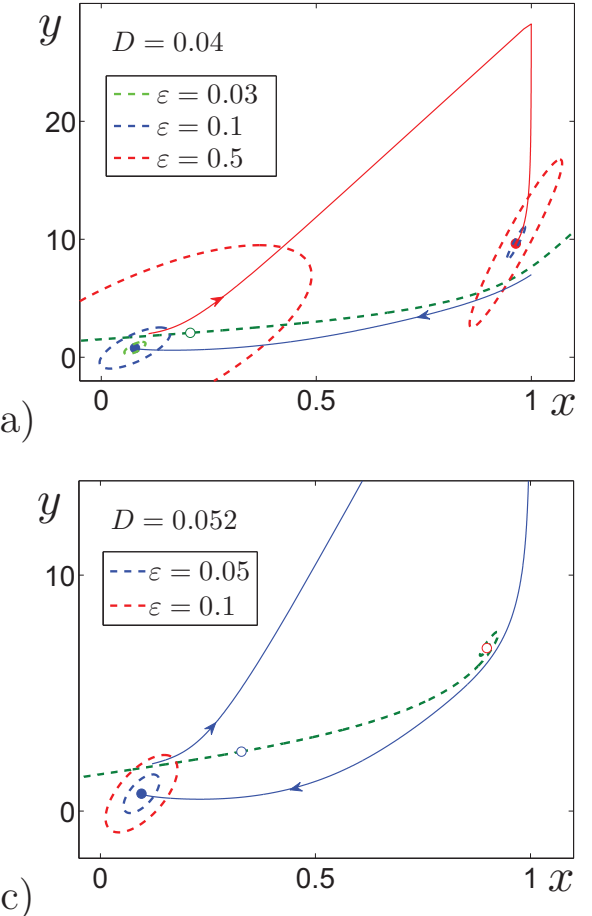

b)
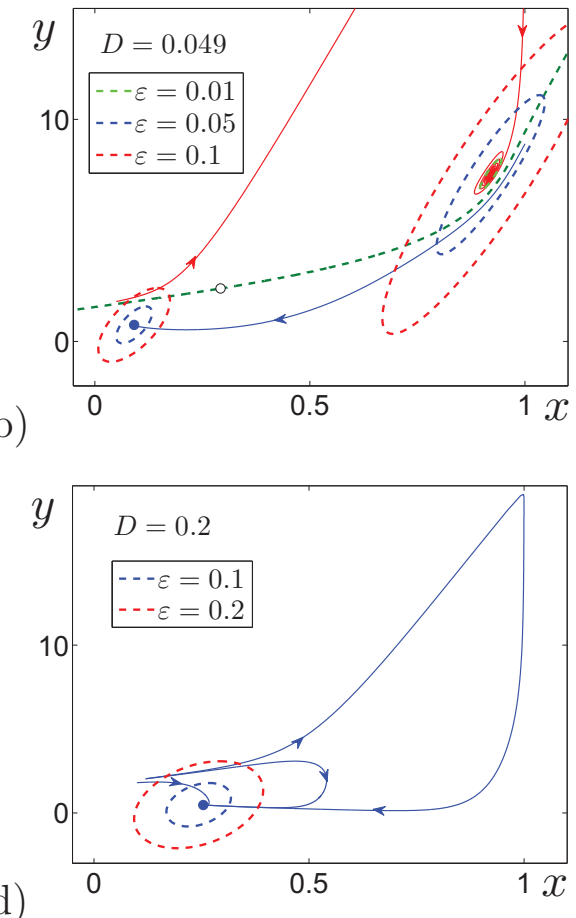

FIG. 8. Confidence ellipses around stable equilibria of system (2) for: (a) $D=0.04$, (b) $D=0.049$, (c) $D=0.052$, and (d) $D=0.2$. arrangement of ellipses and separatrix predicts the onset of noiseinduced transitions from $L$ to $H$. For $\varepsilon=0.5$, both confidence ellipses extend beyond the separatrix. This fact explains bidirectional transitions between $L$ and $S$ and therefore, an appearance multi-modal stochastic oscillations.

In Fig. 8(b), for $D=0.049$ that is closer to the bifurcation point $D_{1}$, for the same $\varepsilon$, confidence ellipses around $H$ are significantly larger than ellipses around $L$. For $\varepsilon=0.05$, the ellipse around $L$ is arranged in the basin of its attraction whereas the ellipse around $H$ intersects the separatrix. This signals about noise-induced transitions from $H$ to $L$. Here, the equilibrium $H$ is more sensitive to noise than $L$ (see Fig. 7 ), so the stochastic system in this bistability regime prefers the equilibrium $L$. For $\varepsilon=0.1$, both ellipses intersect the separatrix, which signals about the stochastic generation of mixed-mode oscillations.
Consider now a case when the stable equilibrium $L$ coexists with the cycle $C$ (see Fig. 9 for $D=0.0506$ ). Here, the equilibrium $L$ is plotted by the blue filled circle, and the stable limit cycle $C$ is shown by the thick red curve. The separatrix $S$ between their basins is plotted by dashed green. In Fig. 9(b), external borders of confidence bands are shown by dashed-dotted lines for two values of the noise intensity. For $\varepsilon=0.0005$, the confidence band entirely belongs to the basin of $C$. Such an arrangement confirms that for $\varepsilon=0.0005$ random trajectories slightly fluctuate near the orbit of the deterministic cycle $C$ [see random trajectories shown in green in Figs. 5(a) and 5(b)]. For $\varepsilon=0.003$, the confidence band partially occupies the basin of the equilibrium $L$ that signals about transitions of random trajectories from $C$ to $L$.

As for opposite transitions from $L$ to $C$, these transitions start to occur for larger noise. Indeed, this fact is confirmed by the a)

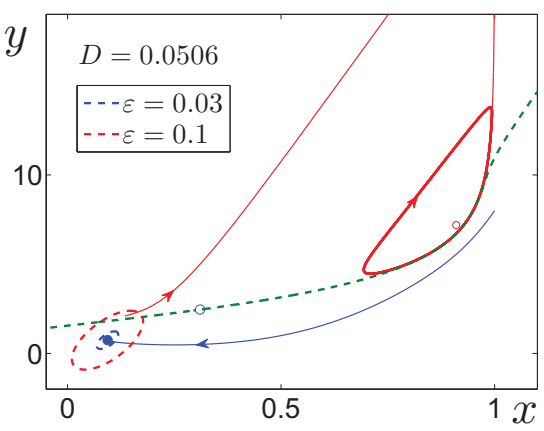

b)

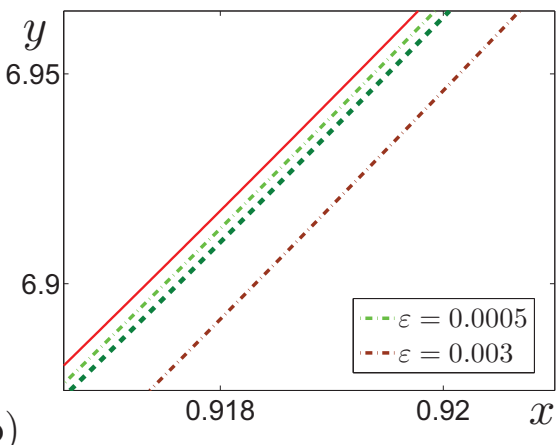

FIG. 9. Confidence domains near attractors of system (2) for $D=0.0506$ : (a) ellipses around the equilibrium $L$ and $(b)$ external borders (dashed-dotted) of confidence bands around the cycle $C$ (thick red). The separatrix is shown by the dashed line. 


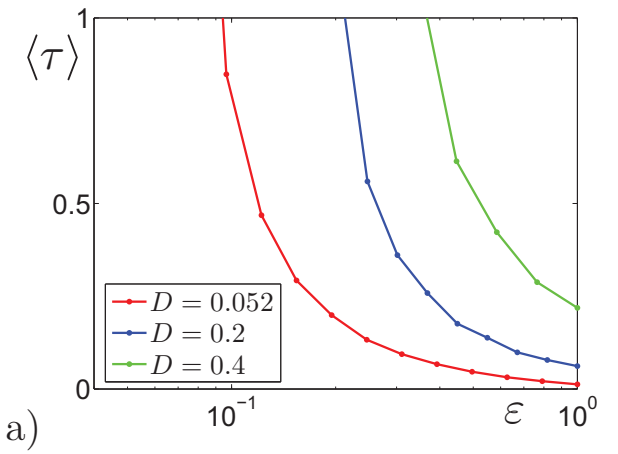

a)

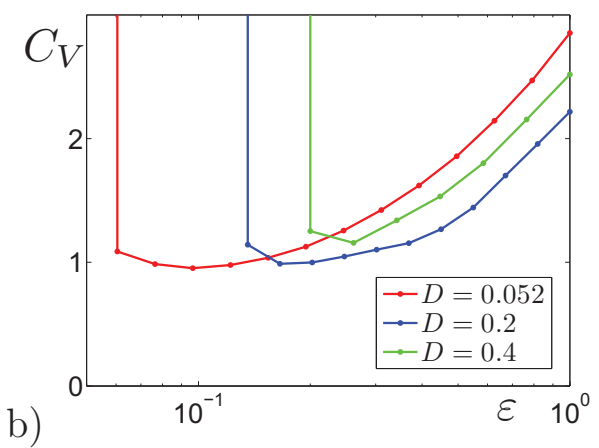

FIG. 10. Statistics of interspike intervals: (a) mean values and (b) coefficients of variation. intersection of the confidence ellipse with the separatrix $S$ [see Fig. 9(a) for $\varepsilon=0.1$. This prognosis well agrees with results of direct numerical simulations in Fig. 5.

Consider now how the method of confidence ellipses works in the analysis of noise-induced excitement in the monostability parameter zone $D>D_{3}$. For this zone, examples are presented in Fig. 8(c) for $D=0.052$ and in Fig. 8 (d) for $D=0.2$. The ellipses show that the onset of the noise-induced excitement under increasing $D$ occurs for larger values of the noise intensity $\varepsilon$.

\section{NOISE-INDUCED SPIKING AND COHERENCE RESONANCE}

As shown above, the phenomenon of noise-induced excitement is accompanied by the appearance of large-amplitude spikes. To describe the frequency properties of spike oscillations, one can use statistics of interspike intervals (ISI). In Fig. 10, mean values $\langle\tau\rangle$ and coefficient of variation $C_{V}$ of the random interspike intervals $\tau$ are plotted for three values of the parameter $D$ vs noise intensity $\varepsilon$.

As can be seen, the onset of the noise-induced excitement is characterized by the sharp drop in mean values of ISI. The further increase of $\varepsilon$ implies the monotonous decrease of these values and some kind of stabilization. A behavior of $C_{V}(\varepsilon)$ is not monotonic. Here, one can see the value of the noise intensity corresponding to the minimum of the variation of ISI. This phenomenon can be classified as coherence resonance. ${ }^{20}$ The coherence resonance is an interesting probabilistic feature of the excitable regime in CSTR.

\section{CONCLUSION}

In this paper, stochastic phenomena in thermochemical kinetics of the continuous stirred tank reactor are studied on the basis of the Zeldovich-Semenov model. Noise-induced complication of dynamical regimes is studied by numerical simulation and analytical approach base on the stochastic sensitivity technique. In bistability zones, stochastic transitions between attractors are studied and the phenomenon of noisy "preference" is explained by the difference in stochastic sensitivity of coexisting attractors. In the monostability zone, noise-induced excitement with generation of large-amplitude spikes as well as the phenomenon of the coherence resonance are demonstrated and discussed.

Although the present paper considers only a separate model of a specific physico-chemical system, the results can be useful in studying a wide range of nonlinear phenomena in real-world complex stochastic processes. Indeed, the stochastic phenomena studied here, related to multistability and excitation, are observed in many dynamical models of complex systems with local and global bifurcations. Therefore, the presented here mathematical technique, based on stochastic sensitivity and confidence domains, has a wide range of applications. An important next step in the development of this technique is the transition from analysis to control problems. Capabilities of the control technique based on the stochastic sensitivity were demonstrated in Ref. 39 for preventing noise-induced ecological shifts and in Ref. 40 for stabilization of the thermochemical system.

\section{ACKNOWLEDGMENTS}

The work was supported by the Russian Foundation for Basic Research (No. 20-01-00165).

\section{DATA AVAILABILITY}

The data that support the findings of this study are available from the corresponding author upon reasonable request.

\section{REFERENCES}

${ }^{1}$ C. van Heerden, "Autothermic processes," Industrial Eng. Chem. 45, 1242-1247 (1953).

${ }^{2}$ D. A. Frank-Kamenetskii, Diffusion and Heat Exchange in Chemical Kinetics (Princeton University Press, Princeton, 1955).

${ }^{3}$ L. D. Schmidt, The Engineering of Chemical Reactions (Oxford University Press, New York, 1998).

${ }^{4}$ M. E. Davis, Fundamentals of Chemical Reaction Engineering (McGraw-Hill, Boston, 2003).

${ }^{5}$ A. Uppal, W. H. Ray, and A. B. Poore, "On the dynamic behavior of continuous stirred tank reactors,” Chem. Eng. Sci. 29, 967-985 (1974).

${ }^{6}$ A. Uppal, W. H. Ray, and A. B. Poore, "The classification of the dynamic behavior of continuous stirred tank reactors-Influence of reactor resident time," Chem. Eng. Sci. 31, 205-214 (1976).

${ }^{7}$ A. L. Kawczyński and J. Gorecki, "Molecular dynamics simulations of sustained oscillations in a thermochemical system," J. Phys. Chem. 96, 1060-1067 (1992).

${ }^{8}$ V. S. Sheplev, S. A. Treskov, and E. P. Volokitin, "Dynamics of a stirred tank reactor with first-order reaction,” Chem. Eng. Sci. 53, 3719-3728 (1998).

${ }^{9}$ A. L. Kawczyński and B. Nowakowski, "Master equation simulations of a model of a thermochemical system," Phys. Rev. E 68, 036218 (2003).

${ }^{10}$ V. I. Bykov, S. B. Tsybenova, and G. Yablonsky, Chemical Complexity via Simple Models (De Gruyter, Berlin, 2018). 
${ }^{11}$ D. A. Vaganov, N. G. Samoilenko, and V. G. Abramov, "Periodic regimes of continuous stirred tank reactors," Chem. Eng. Sci. 33, 1131-1140 (1978).

${ }^{12} \mathrm{~V}$. I. Bykov and S. B. Tsybenova, "Parametric analysis of the simplest model of the theory of thermal explosion-The Zel'dovich-Semenov model," Combust. Explos. Shock Waves 37, 523-534 (2001).

${ }^{13}$ V. I. Bykov, L. A. Serafimov, and S. B. Tsybenova, "Emergency starting regimes of a continuous stirred tank reactor," Theoret. Found. Chem. Eng. 49, 361-369 (2015)

${ }^{14} \mathrm{~N}$. N. Semenov, "Theories of combustion processes," Z. Phys. Chem. 48, 571-582 (1928).

${ }^{15} \mathrm{~J}$. B. Zeldovich and J. A. Zysin, "On the theory of heat stress. Exothermic reaction in the jet," Z. Tekhn. Fiz. 11, 501-508 (1941).

${ }^{16} \mathrm{~W}$. Horsthemke and R. Lefever, Noise-Induced Transitions (Springer, Berlin, 1984).

${ }^{17}$ V. S. Anishchenko, V. V. Astakhov, A. B. Neiman, T. E. Vadivasova, and L. Schimansky-Geier, Nonlinear Dynamics of Chaotic and Stochastic Systems. Tutorial and Modern Development (Springer-Verlag, Berlin, 2007).

${ }^{18}$ M. D. McDonnell, N. G. Stocks, C. E. M. Pearce, and D. Abbott, Stochastic Resonance: From Suprathreshold Stochastic Resonance to Stochastic Signal Quantization (Cambridge University Press, 2008).

${ }^{19}$ L. Gammaitoni, P. Hanggi, P. Jung, and F. Marchesoni, "Stochastic resonance," Rev. Mod. Phys. 70(1), 223-287 (1998).

${ }^{20}$ A. S. Pikovsky and J. Kurths, "Coherence resonance in a noise-driven excitable system," Phys. Rev. Lett. 78, 775-778 (1997).

${ }^{21}$ B. Lindner, J. Garcia-Ojalvo, A. Neiman, and L. Schimansky-Geier, "Effects of noise in excitable systems," Phys. Rep. 392, 321-424 (2004).

${ }^{22}$ J. B. Gao, S. K. Hwang, and J. M. Liu, "When can noise induce chaos?," Phys. Rev. Lett. 82(6), 1132-1135 (1999).

${ }^{23}$ Y.-C. Lai and T. Tel, Transient Chaos. Complex Dynamics on Finite Time Scales (Springer-Verlag, New York, 2011).

${ }^{24}$ I. Bashkirtseva, L. Ryashko, and E. Slepukhina, "Noise-induced oscillation bistability and transition to chaos in FitzHugh-Nagumo model," Fluctuat. Noise Lett. 13(1), 1450004 (2014).

${ }^{25}$ L. Arnold, Random Dynamical Systems (Springer-Verlag, Berlin, 1998).
${ }^{26}$ M. A. Zaks, X. Sailer, L. Schimansky-Geier, and A. B. Neiman, "Noise induced complexity: From subthreshold oscillations to spiking in coupled excitable systems," Chaos 15(2), 026117 (2005).

${ }^{27}$ M. I. Freidlin and A. D. Wentzell, Random Perturbations of Dynamical Systems (Springer-Verlag, New York, 1984).

${ }^{28} \mathrm{C}$. W. Gardiner, Handbook of Stochastic Methods for Physics, Chemistry and the Natural Sciences (Springer-Verlag, Berlin, 1983).

${ }^{29}$ I. A. Bashkirtseva and L. B. Ryashko, "Sensitivity analysis of stochastically forced Lorenz model cycles under period doubling bifurcations," Dyn. Syst. Appl. 11(2), 293-310 (2002).

${ }^{30}$ I. Bashkirtseva, L. Ryashko, and P. Stikhin, "Noise-induced backward bifurcations of stochastic 3D-cycles," Fluctuat. Noise Lett. 9, 89-106 (2010).

${ }^{31} \mathrm{~J}$. Jungeilges, T. Ryazanova, A. Mitrofanova, and I. Popova, "Sensitivity analysis of consumption cycles," Chaos 28(5), 055905 (2018).

${ }^{32}$ I. Bashkirtseva, "Stochastic sensitivity of systems driven by colored noise," Physica A 505, 729-736 (2018)

${ }^{33}$ I. Bashkirtseva, G. Chen, and L. Ryashko, "Analysis of stochastic cycles in the Chen system,” Int. J. Bifurcat. Chaos 20(05), 1439-1450 (2010).

${ }^{34}$ I. Bashkirtseva, G. Chen, and L. Ryashko, "Analysis of noise-induced transitions from regular to chaotic oscillations in the Chen system," Chaos 22, 033104 (2012).

${ }^{35}$ E. S. Slepukhina, "Sensitivity analysis of noise-induced mixed-mode oscillations in Morris-Lecar neuron model," Math. Model. Nat. Phenom. 12(4), 74-90 (2017).

${ }^{36} \mathrm{~L}$. Ryashko, "Sensitivity analysis of the noise-induced oscillatory multistability in Higgins model of glycolysis," Chaos 28(3), 033602 (2018).

${ }^{37}$ I. Bashkirtseva, "Crises, noise, and tipping in the Hassell population model," Chaos 28, 033603 (2018).

${ }^{38} \mathrm{~A}$. Belyaev and T. Ryazanova, "Stochastic sensitivity of attractors for a piecewise smooth neuron model,” J. Differ. Eqs. Appl. 25, 1468-1487 (2019).

${ }^{39} \mathrm{~L}$. Ryashko and I. Bashkirtseva, "Stochastic sensitivity analysis and control for ecological model with the Allee effect," Math. Model. Nat. Phenom. 10(2), 130-140 (2015).

${ }^{40}$ I. Bashkirtseva, "Controlling the stochastic sensitivity in thermochemical systems under incomplete information," Kybernetika 54, 96-109 (2018). 